\title{
REVIEW OF METHODS FOR DETERMINING THE SPATIAL RESOLUTION OF UAV SENSORS
}

\author{
A. Orych ${ }^{a *}$ \\ ${ }^{\text {a }}$ Department of Remote Sensing and Photogrammetry, Geodesy Institute, Faculty of Civil Engineering and Geodesy, Military \\ University of Technology, Warsaw, Poland - agata.orych@wat.edu.pl
}

KEY WORDS: UAV, Calibration target, GRD, Spatial resolution, Siemens Star

\begin{abstract}
:
The ground resolved distance (GRD) of an imaging sensor, i.e. the size of the smallest element distinguishable on acquired imagery, is one of the most important sensor quality assessment factors, as it is directly linked to the amount of information that can be derived from the end product. The paper is a review of a wide variety of calibration targets used for determining the spatial resolution of remote sensing sensors. The author provides a description of calibration targets used historically and then moves on to high-frequency targets used for high-resolution remote sensing imaging equipment. As analysis is made which of these types of targets are best suited for UAV sensors, taking into account parameters very specific to UAVs: frame size, small GSD values and low flight stability.
\end{abstract}

\section{INTRODUCTION}

The spatial resolution of a sensor describes its geometrical quality and determines what type of objects it is able to image correctly. The spatial resolution of a sensor is defined by two measures - ground sampling distance (GSD) and ground resolved distance (GRD). The ground sampling distance is the distance, in field units, between the centres of two neighbouring detectors (i.e. two neighbouring pixels on the image). When working with an ideal sensor it's GSD is equal to the inverse of the Nyquist frequency for that detector array. However, the GSD of a sensor is simply a theoretical measure, which only takes into account the geometry of the detector elements and the distance between it and the imaged objects. The actual resolution of the sensor is highly dependent on other limiting factors of the imaging system, such as interior noise, the parameters of the lossless analogue-to-digital conversion of the signal and the system optics, which never have a $100 \%$ transmission and usually introduce additional artefacts.

The ground resolved distance of a sensor is determined on the basis of visual analyses of specially constructed calibration targets (Keating, 1997). These targets can take on a number of different forms (eg a Siemens Star or bar target), sizes and can be constructed using a variety of different materials.

\section{CALIBRATION TARGETS}

\subsection{Definition}

The term calibration target is an especially designed structure, which when laid out flat on the ground and photographed, can be used to evaluate the sensor's imaging capabilities. Each target has very specific geometrical and spectral characteristics. Such structures usually have a flat modular design with the geometrical calibration elements applied with paints. These elements have very carefully designed spatial-frequency characteristics. Determining the ground resolved distance is based on analyses of images of these elements in terms of the amount of information (details) which had been transferred from the actual target, via the atmosphere, the optical system and imaging system, to the final image.

\subsection{Types of calibration targets}

There is a great number of calibration targets used, their size and shape depending on their expected use. Some of the most popular calibration targets include:

$\begin{array}{ll}- & \text { EIA } 1956 \text { Test Pattern } \\ \text { - } & \text { USAF } 1951 \text { Test Pattern } \\ \text { - } & \text { Bar Target } \\ \text { - } & \text { ISO } 12233 \text { Test Pattern } \\ \text { - } & \text { Norman Koren Target } \\ \text { - } & \text { Slanted Edge Test } \\ & \text { Siemens Star Test }\end{array}$

The beginnings of resolution testing of photographic systems coincides with the beginnings of using such sensors to acquire imagery for purposes, in which the spatial resolution is an important factor. Such applications include photogrammetry and military reconnaissance. This can be dated back to the beginning of the twentieth century. However, the development of methods for determining the resolving power of digital optical devices (used both for recording and displaying imagery data) can already be seen in the 1950 's, with the advent of the television.

Tests such as the EIA 1965 (Figure 1), which was used to assess the quality of the image displayed on the screens of black-andwhite televisions, are the basis for many test patterns used today to study modern digital sensors.

* Corresponding author 


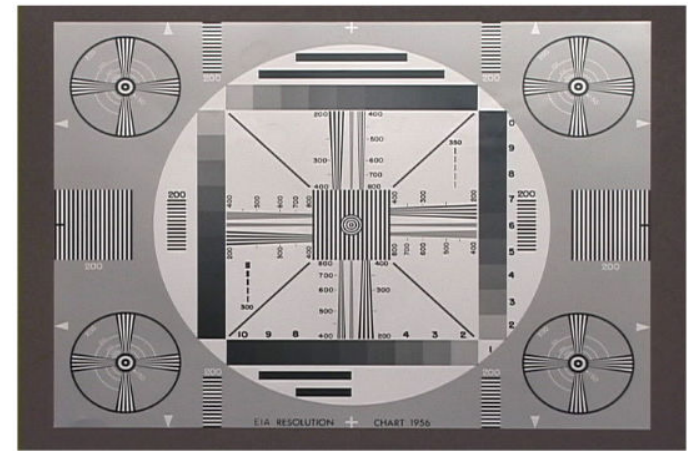

Figure 1. EIA 1956 Test Pattern (Messina, 2006)

The EIA Test Pattern was used to determine the quality of a monitor in units of the ratio between the number of visible lines per picture height. The basic elements which allow for such measurements are the narrow wedges of different widths visible in Figure 1. Analyzing the image staring with the thickest lines (i.e. cycles with the lowest frequencies) and continuing to the high-frequency cycles, the moment at which the lines at a given frequency become impossible to distinguish determines the resolving power of the optical system (Task, 1979). The process of image analysis is a subjective process because it is based on an individual interpretation by the observer regarding the definition of what situation on the image will be deemed resolved (distinguishable) and what will not. In addition, the results of such an analysis depends largely on the state of the observer's visual system. The 1956 EIA Test Pattern was used to assess television monitors in the mid XXth century. These devices which were characterized by a resolution from 300 to 480 line widths per picture width (LW / PW). With advances in technology, a significant increase in the image quality of household TV systems (eg. HD TVs with a resolution equal to $1018 \mathrm{LW} / \mathrm{PW}$ ) and the use of digital technologies for other applications (eg. in framing cameras), the need arose to develop further types of calibration tests, to be used with these relatively high quality devices.

To test the quality of sensors mounted on UAV's, night vision goggles and other imaging devices, the United States Air Force use the USAF 1951 Test Pattern.

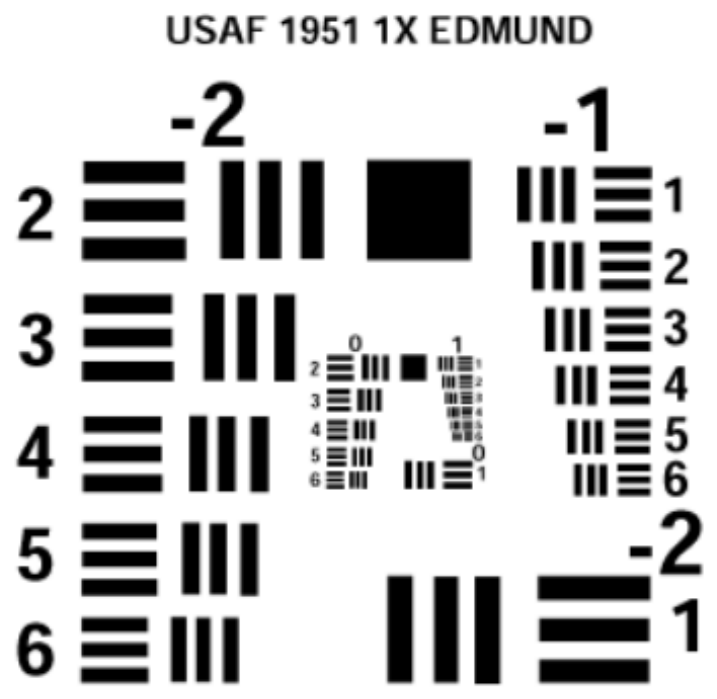

Figure 2. USAF 1951 Test Pattern (Messina, 2006)
The USAF 1951 Test Pattern is a tri-bar target. The scale factor between consecutive bar groups is fixed and equal to the sixth root of two. Due to its size and relatively complex design, this target is mainly used for laboratory tests (Messina, 2006). Resolution is determined in a similar way as was the case with the EIA 1956 test - by assessing the visibility of groups of bars and identifying the smallest group for which all three black bars can still be separated.

Many different types of bar targets for use in field conditions have been developed based on the principles of the USAF1951 test. An example of such a test is shown in the following figure.

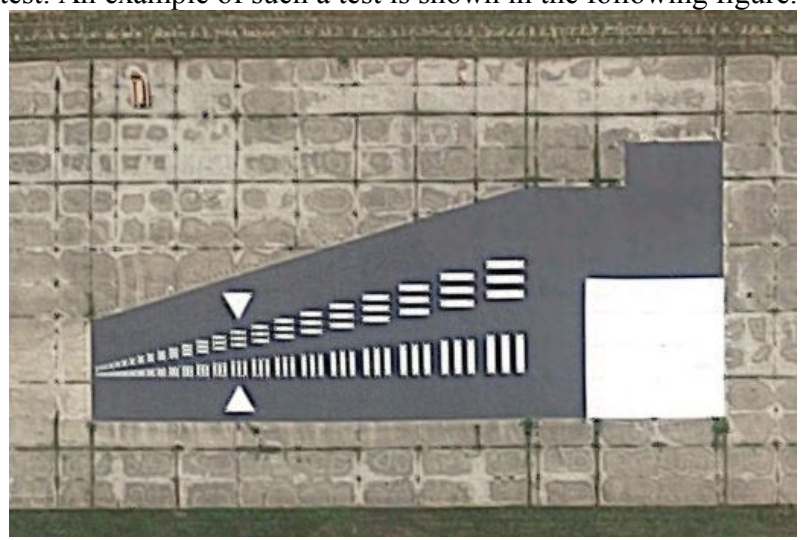

Figure 3. Bar target calibration test at Wright Patterson OSMPF, USA. (Google Earth)

The calibration target presented in Figure 3 is a bar target located at Wright Patterson OSMPF (Open Skies Media Processing Facility), USA. Such bar targets can consist of the bi-bar and tri-bar components or more. According to (FLIR92, 1993) and (Osterberg, 1962) RGB sensors are usually tested using tri-bar tests whilst near-infrared and thermal sensors should be analysed using 4-bar targets. In the illustrated example, each group consists of three vertical bars and three horizontal bars. Each bar has a height equal to five times their width. The dimensions of the bars in successive groups have a ratio equal to the sixth root of two, similarly to the previously described test pattern. The size range of all bar groups depends on the imaging capabilities of the tested sensor.

There are many different types of bar targets for testing digital sensors under different conditions, for example the Norman Koren Test.

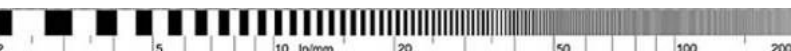

Figure 4. Norman Koren Test. (www.normankoren.com)

The test takes the form of alternately arranged black-and-white bars with a gradually increasing frequency. It is used for determining the resolving power of sensor optics and lightsensitive materials giving results from 2 to 200 line pairs per millimetre.

In 2000 a new test pattern was developed together with a detailed description of the methodology for determining the quality parameters of high resolution sensors, including HDTV cameras. In accordance with its title: "Electronic still picture imaging - Resolution and spatial frequency responses," the ISO 12233 Test Pattern is an international standard for measuring the spatial resolution and frequency of electronic photographic 
systems. The test was designed in a way that gives the possibility to determine resolutions up to $4000 \mathrm{LW} / \mathrm{PW}$. The test allows not only the analysis of image sharpness but also the evaluation of distortion and chromatic aberration.

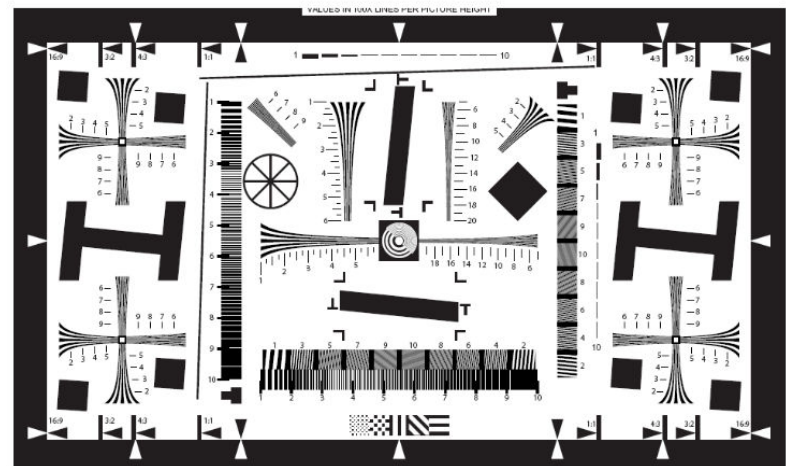

Figure5. ISO 12233 Test Pattern (ISO-12233:2014)

The ISO12233 Test Pattern consists of the already familiar wedges from the EIA1965 test and bar groups from the USAF1951 target. The board also includes a number of diagonal elements which form the Slanted Edges.

A Slanted Edge Test usually takes on the form of a black square (or one of its edges) on a white background that is rotated relative to the vertical by a few degrees as seen in Figure 6 .

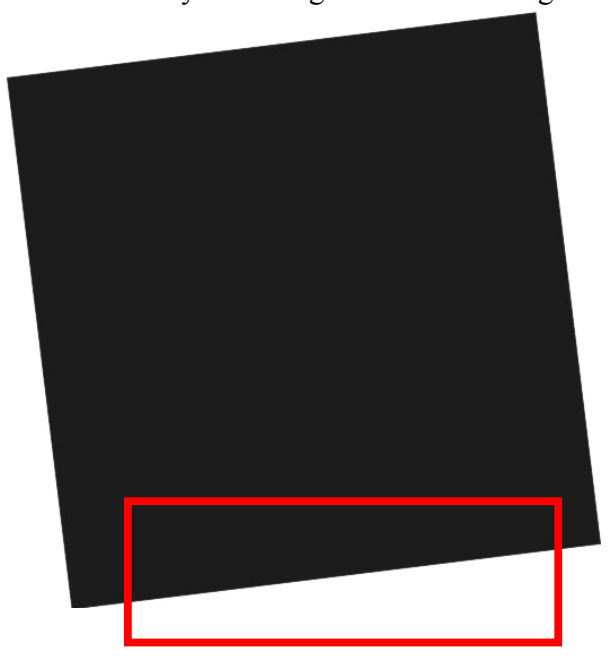

Figure 6. Slanted Edge Test

Evaluation of image resolution using this type of target is only possible through the use of appropriate measurement tools, as there is no way to determine the resolution of the Slanted Edge Test based only on a visual analysis of the image.

The test shown in Figure 7 is a Siemens Star. This target takes on a circular shape with alternatively arranged contrasting sectors with a constant angle of $\theta$. The number of sectors may vary, giving different levels of accuracy. At the outer edge of the star, the black and white sectors have a very low-frequency (the sectors are wide). Along with a decrease in the length of the radius from the centre of the Star, the spatial frequency of the target components increases. As in the case of bar targets, when increasing the frequency between the target elements, the image of these elements will firstly become slightly defocused (decreasing the amplitude of intensities of the black and white elements), until the moment in which the amplitude between the contrasting elements falls to zero and the elements will impossible to distinguish. By determining the star radius at which this phenomenon occurs, it is possible to calculate the ground resolved distance of the imaging sensor.
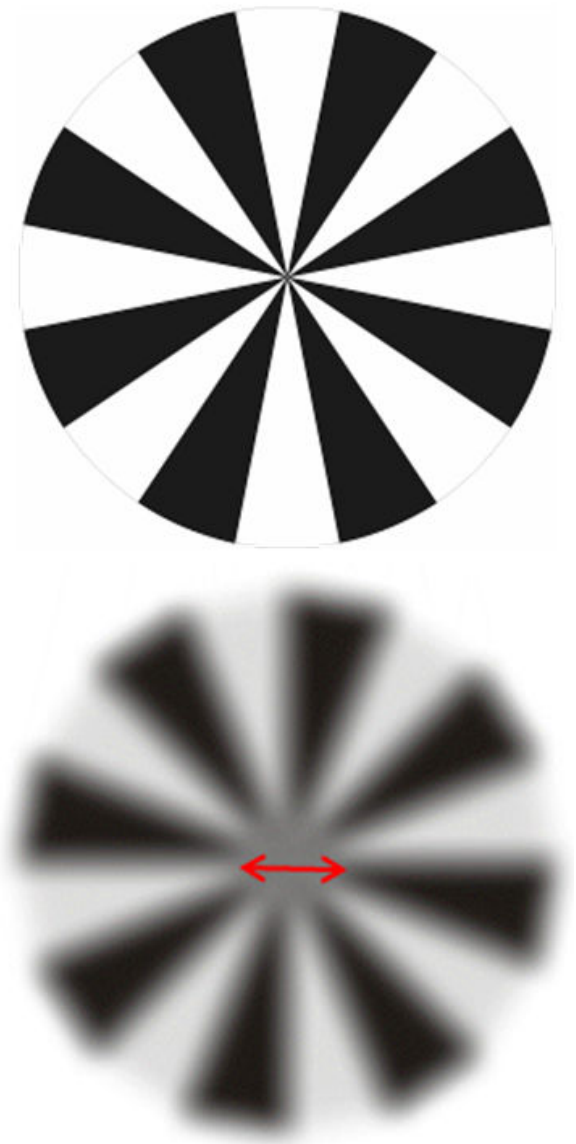

Figure 7. Siemens star Target in its theoretical form (top image) and imaged (lower image) with the blurred area visible in the centre.

Depending on the intended use of the target, there are two types of Siemens Stars used - a sinusoidal test pattern is used in laboratory measurements (Loebich, et.al, 2007) whereas a binary (discrete) Star, as the one shown above, is used for outdoor testing (Honkavaara et al., 2008).

\section{CHOICE OF CALIBRATION TARGET FOR EVALUATING UAV SENSORS}

Based on a preliminary analysis, three types of test patterns were selected as possible choices for evaluating the quality of imagery acquired by UAV sensors: bar target, Slanted Edge Test and Siemens Star.

One of the advantages of using a conventional bar target for testing digital UAV sensor systems is it's very simple geometry which makes it easy to design and build. Visual analysis of the images is also easy and requires the observer only to indicate the smallest group of bars that are possible to resolve. However, this method is completely subjective and, unfortunately, the design of the test pattern makes it impossible to automate the process of determining the ground resolution, without major changes to its structure or very complex calculation algorithms. Additionally, because of such a design, GRD measurements based on a single image can only be done in a discrete manner. Existing methodologies for determining the spatial resolution of digital optical sensors, usually require a large number of flights 
over the target and a large number of observations made by a group of independent observers. This methodology gives very high accuracies, but at the expense of large amounts of data and thus is very time-consuming and expensive.

The Slanted Edge Test is also a very popular test pattern used for evaluating digital aerial sensors. It has a very simple design, which makes it possible to determine the quality of the imaging system automatically, and therefore objectively, which makes it very popular with laboratory testing. However, one of the main disadvantaged of using this test is that it is impossible to determine resolution without the support of computer tools and advanced mathematical algorithms in the frequency domain (Zhang et al., 2012). Additionally, this type of target and the algorithms used for the analysis, are not well suited for highly aliased imagery, which is often the same with light-weight, lowcost video sensors used on UAV platforms (www.imatest.com.).

The calibration target, which seems to meet all the requirements for testing digital sensors for UAVs, is a Siemens Star. Compared to the previously described test patterns, the Siemens Star has a relatively more complex geometry, but thanks to this design, gives an opportunity to analyze resolution in all directions with respect to the flight path of the aircraft. When using this target, it is possible to define resolution in a continuous manner, being only limited by the chord length at the edge of each sector. The analysis of spatial resolution based on the Star Siemens requires the use of additional measurement tools (e.g. a measuring tool in any basic graphics software), however, it is possible to perform this measurement both measurement subjectively and objectively. When working binary Siemens Stars, for assessing UAV systems in nonlaboratory conditions, the CFT (Contrast Transfer Function) can be used to easily automate this process (Reulke et al., 2006). According to Imatest, a leading manufacturer of test patterns and software for image quality analyses, this type of target is indifferent to the negative effects of image aliasing, and on attempts to artificially improve image resolution through sharpening or noise reduction.

\section{OTHER APPLICATIONS OF SIEMENS STAR TARGETS}

Due to its advantages, Siemens Stars are widely used in many fields that use imaging sensors such as examining holographic sensors (Stockmar, et al., 2013) or in the field of X-ray imaging (Hofsten, et al., 2007). An interesting application of this type of target is to study so-called. Depth cameras. These are cameras based on ToF (Time of Flight) technology i.e. they measure the movement of each point on the scene This technology is often compared LiDAR technology, except that the data are not obtained point by point with an active sensor, but are recorded at the same time for the entire scene. This technology is used in many everyday applications, for example in active pedestrian safety (Hsu, et al, 2006), or as an additional element in the latest game consoles such as the Xbox One, allowing the user greater interaction in many games (Rubin, 2013). The technology is also used for topographical applications for obtaining digital elevation models (Nitsche et al, 2013).

The Siemens Star design was also used as a basis for a new type of calibration target used to study laser scanners used in terrestrial photogrammetry.

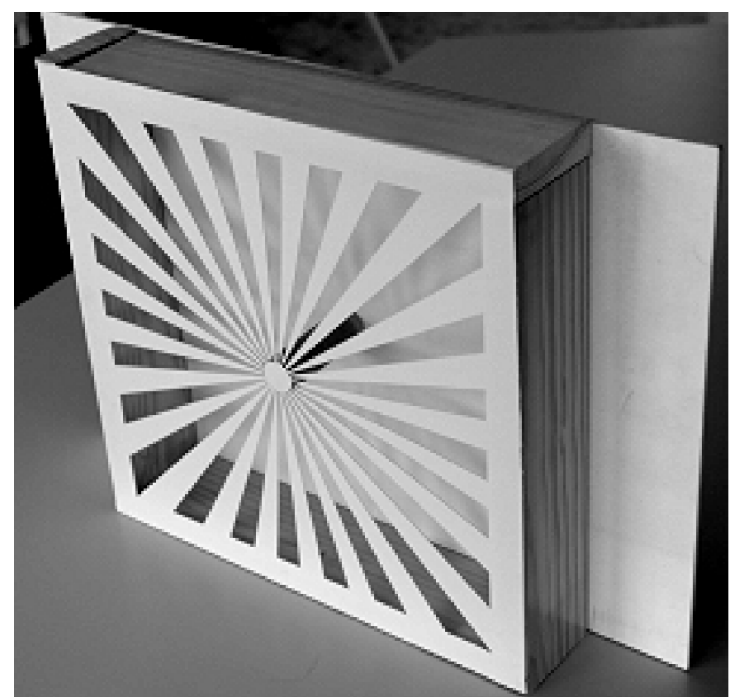

Figure 8. Boehler Star used to measure the spatial resolution of laser scanners (Boehler, et al., 2003)

Figure 8 shows the so-called Boehler Star, which is a threedimensional form of the Star Siemens for determining the spatial resolution laser scanner sensors. If the tested sensor has a high resolution, the resulting point cloud should consist not only of points on the front wall of the target, but also include points on the back wall of the target. The better the resolution of the tested sensor, the more points on the back wall will be visible close to the centre of the. (Boehler, et al., 2003)

\section{CONCLUSION}

When planning UAV image acquisition missions, the most important image quality factor which must be taken into account, is the spatial resolution of the acquired imagery. This parameter dictates the flying altitude and sensor exposure parameters. An analysis of a series of test patterns has shown, that a binary Siemens Star is the best choice when examining the capabilities of digital aerial sensors. Such a target is ideal for use with UAV sensors as it does not require flights to be conducted precisely in a specific direction or orientation relative to the target. This is especially useful when working with non-fixed-wing UAV's. A Siemens Star can be used to determine the resolution in all directions and in a fast, objective manner. Due to its design, the Siemens Star, in comparison to the most commonly used bar targets, has relatively small dimensions, which is ideal for low altitude imaging from UAV platforms.

\section{ACKNOWLEDGEMENTS}

Research presented in this paper was conducted as part of the project: "IRAMSWater - Innovative remote sensing system for the monitoring of pollutants in rivers, offshore waters and flooded areas" (PBS1/B9/8/2012) financed by the polish National Centre for Research and Development. The main objective of this project, is to create a remote sensing system based on hyperspectral sensors which will enable the evaluation, detection and distribution of biological, physical and chemical pollutants in the examined waters in real time. 


\section{REFERENCES}

Boehler W., Bordas V.M., Marbs A., "Investigating Laser Scanner Accuracy", XIXth CIPA Symposium, Turkey, 2003

FLIR92. "Thermal Imaging Systems Perfomance Model Analyst's Reference Guide". USA: US Army Night Vision and Electronic Sensors Directorate, 1993.

Hofsten O., Takman P., Vogt U., "Simulation of partially coherent image formation in a compact soft X-ray microscope", Ultramicroscopy, Elsevier, 2007

Honkavaara, E., Peltoniemi, J., Ahokas, E., Kuittinen, R., Hyyppä, J., Jaakkola, J., Kaartinen, H., Markelin, L., Nurmine, K., Suomalainen, J., "Permanent Test Field for Digital Photogrammetric Systems", Photogrammetric Engineering \& Remote Sensing, Number 1, 2008

Hsu, S., Acharya, S., Rafii, A., New, R., "Performance of a Time-of-Flight Range Camera for Intelligent Vehicle Safety Applications", Advanced Microsystems for Automotive Applications, Springer, 2006.

ISO-12233 "Photography -- Electronic still picture imaging -Resolution and spatial frequency responses.", 2014

Loebich C., Wueller D., Klingen B., Jaeger A., "Digital camera resolution measurement using sinusoidal Siemens Stars", Proc. SPIE 6502, Digital Photography III, 2007

Messina, E. "Standards for visual acuity." ASTM International Task Group on Performance Measures for Robots for Urdan Search and Rescue. 2006.

Nitsche, M., Turowski, J. M., Badoux, A., Rickenmann, D., Kohoutek, T. K., Pauli, M., Kirchner, J. W., "Range imaging:
A new method for high-resolution topographic measurements in small-and medium-scale field sites" , Earth Surface Processes and Landforms, 2013

Osterberg, H. "Military Standardization Handbook - Optical Design, Evaluation phase optical tests." USA: Defence Supply Agency, 1962.

Reulke R., Becker S., Haala N., Tempelmann U. "Determination and improvement of spatial resolution of the CCD-line-scanner system ADS40", ISPRS Journal of Photogrammetry and Remote Sensing, Volume 60, Issue 22006

Rubin P. "Exclusive First Look at Xbox One" Wired Magazine. - 2013.

Stockmar, M., Cloetens, P., Zanette, I., Enders, B., Dierolf, M., Pfeiffer, F., Thibault, P., "Near-field ptychography: phase retrieval for inline holography using a structured illumination", Scientific Reports., 2013

Task, H. L. "An evaluation and comparison of several measures of image quality for television displays." USA: Write Patterson Airforce Base, 1979.

Zhang X., Kashti T., Kella D., Frank T., Shaked D., Ulichney R., Fischer M., Allebach J.P., "Measuring the Modulation Transfer Function of Image Capture Devices: What Do the Numbers Really Mean?" Proc. SPIE 8293, Image Quality and System Performance IX, 2012

http://www.normankoren.com/Tutorials/MTF5.html.

http://www.imatest.com 